

(REVIEW ARTICLE)

\title{
Postpartum depression and the scarcity of generalizable evidence: A mini-review of existing literature and the way forward
}

\author{
Fidelis E Uwumiro ${ }^{1, *}$, Victory 0 Okpujie ${ }^{2}$ and Kingsley 0 Anokwuru 1 \\ ${ }^{1}$ Our Lady of Apostles Hospital, Akwanga, Nasarawa State, Nigeria. \\ ${ }^{2}$ Central Hospital Benin, Benin city, Edo State, Nigeria.
}

World Journal of Advanced Research and Reviews, 2021, 11(02), 081-084

Publication history: Received on 30 June 2021; revised on 04 August 2021; accepted on 06 August 2021

Article DOI: https://doi.org/10.30574/wjarr.2021.11.2.0364

\begin{abstract}
The first known reference to Postpartum Depression (PPD), was Hippocrates' 4th century hypothesis that drainage of lochia, if suppressed, could flow to the brain resulting in agitation, delirium, and episodes of mania. This hypothesis became dogma and lasted over a millennium. Over the years, knowledge of PPD has evolved but researchers still struggle to establish it as a distinct disease entity. It was initially recognized as Major Depressive Disorder (MDD) with postpartum onset in the 1994 revision of the Diagnostic and Statistical Manual of Mental Disorders (DSM-IV), the "bible" of diagnostic criteria for mental health professionals and researchers. It is currently recognized as MDD with peripartum onset (DSM-V) and researchers have admitted that persuasive evidence to indicate that postpartum depression is distinct from other existing depressive disorders, has not been found. Several diagnostic tools such as the Edinburg Postpartum Depression Scale (EDPS), the Beck Depression Inventory (BDI-II), Patient Health Questionnaire (PHQ-9), and the Postpartum Depression Screening Scale (PDSS) are available for use but none of these is universally accepted. With much of the published literature plagued with bias and structural inconsistencies, the credibility of the evidence has been greatly diminished. The difficulty with disease designation, the absence of a universally accepted tool for diagnosis and the scarcity of generalizable evidence on the subject has impaired the early recognition and effective management of PPD. It is therefore expedient, to critically appraise some of the available literature and proffer solutions to navigating this conundrum.
\end{abstract}

Keywords: Postpartum depression; Perinatal depression; Infanticide; Depressive disorders; Depression in pregnancy

\section{Introduction}

Postpartum depression (PPD) has been described as the most common psychiatric complication among women of childbearing age [1]. PPD can present abruptly after delivery or evolve insidiously through pregnancy. It has been reported by the World Health Organization (WHO) to occur in about $10 \%$ of pregnant women worldwide and in up to $10 \%-15 \%$ of women who have just given birth. It has also been reported in at least 1 out of 8 women in the United States [2]. PPD has been classified by the Diagnostic and Statistical Manual of Mental Disorders (DSM-5), as any episode of major depressive disorder that begins within four weeks of childbirth and presents with persistent feelings of sadness or hopelessness, fatigue, loss of interest, poor sleep (even when the baby is sleeping), trouble bonding with the baby and thoughts of death, suicide or harming oneself and the baby (National Institute of Mental Health, 2020). Despite its wide recognition, the cause of PPD remains unknown but women with a combination of genetic and environmental factors such as major life stressors, previous history of depression or anxiety, risky pregnancy and mode of delivery like emergency cesarean delivery and hospitalization have been associated with higher rates of PPD. Also, factors such as inability to lactate or breastfeed, young maternal age, absence of social, emotional and financial support, domestic violence, poor exercise/diet and a sick baby have been associated with higher incidence of PPD [3].

\footnotetext{
* Corresponding author: Fidelis E Uwumiro

Our Lady of Apostles Hospital, Akwanga, Nasarawa State, Nigeria.
}

Copyright (c) 2021 Author(s) retain the copyright of this article. This article is published under the terms of the Creative Commons Attribution License 4.0. 
PPD is estimated to affect $20 \%-40 \%$ of women living in low-income countries, with higher incidence in those with unplanned pregnancies, lack of partner support and maternal illness after delivery [4]. While the prevalence of PPD has remained difficult to estimate due to variations in diagnostic criteria, under-reporting by mothers themselves and failure of care provider to probe for symptoms and signs of PPD appears to contribute to its prevalence. Available evidence suggests that only $20 \%$ of women who experience symptoms of PPD actually report them. Also, the tendency of mothers, care providers and society to view these symptoms as normal natural consequences of child-birth only worsens the prevalence [5]. Many women for fear of being seen as bad mothers will not report symptoms of PPD at all [6].

\section{Review of existing literature}

Despite the widespread reports and the growing literature suggesting the global burden of PPD, general knowledge about it is still poor and it continues to be overlooked in clinical practice [7]. The effects of PPD clearly go beyond the mother to affect her baby and partner and the emerging picture is that the incidence of PPD may be higher in reality than currently reported because not all women are assessed for the condition and not all women assessed are effectively treated [8]. These issues, combined with the risk of suicide, infanticide, poor breastfeeding and its complications, make PPD a serious social and public health problem. It is necessary then to appraise critically, the merits of its burden, and examine some of the available knowledge on the best options for mitigating risk among patients with PPD.

In 2005, a group of researchers (Gavin et al.) embarked on a systematic review of the evidence on the incidence and prevalence of postpartum depression with the aim of comparing results with those of depression in women at nonchildbearing times. They drew data from multiple online sources (including MEDLINE, CINAHL, PsycINFO and Sociofile) spanning a period of 24 years (1980 - 2004) and combined those with hand-searched bibliographies and consultations with professionals. Their method was to combine the strength of the evidence from multiple cross-sectional, cohort and case-control studies with that of structured clinical interviews through a meta-analysis. Their aim, was to test the merit of their assumption that there was at least some difference in PPD rates during childbearing and non-childbearing times. Of 109 articles reviewed, 28 met the criteria for inclusion. Results showed $6.5 \%-12.9 \%$ combined point prevalence of PPD in different trimesters and months in the first postpartum year. Period prevalence for the study was put at $19.2 \%$. The objective of this study would have been achieved by conducting separate meta-analyses of data among women in peripartum period and comparing results with those in non-childbearing period. While the data sources from this study may have been reflective of women in developed countries, no attempt was made to incorporate studies from lowincome countries. Also, the inclusion of largely hospital data and the opinion of professionals suggests critical selection bias in this study. The study showed no evidence of measures implemented to control this bias. Furthermore, it is not clear if sub-populations of women (for example those who had pre-pregnancy depression) were included or excluded from the study. It is also not clear whether temporality was established for the included cohort studies. These flaws, combined with a meagre 28 article-sized meta-analysis and a wide 95\% confidence interval, may have compromised the external validity of the study and created significant uncertainty as regards the actual levels of PPD. Ultimately, the objective of the study could not be achieved, a point Gavin and colleagues note well in their conclusions.

In a recent contrast to the study above, another group of inquisitors (Shittu et al. 2019) recruited 596 postpartum mothers from rural Ethiopia in a single-staged cluster sampling, to assess the prevalence and associated factors of PPD among women who gave birth in the preceding twelve months in low-income areas. Data was collected with interviewer-administered questionnaire and PPD was diagnosed using standardized scales. With a response rate of 97.4\%, PPD prevalence was $23.7 \%$ (95\% CI: 20.3 - 27.2). The study also identified marital status, unwanted pregnancy, unwanted infant sex, infant illness and low social support as independent predictors of postpartum depression. The study design and population, though appropriate for the objectives, does not provide an opportunity for contrast with women from developed countries or with women from better social strata in the same environment. Also, the exclusion of pregnant women from the study denies us insights into the evolving nature of PPD. Further, seriously ill hospitalized women and those who gave birth less than 2 weeks before the study (some of whom may be showing early signs of PPD) were excluded, again depriving us of critical insights into the effects of illness and hospitalization on the prevalence of PPD. Perhaps researchers thought it was only fair not to add to the burden of these women. Furthermore, women with borderline scores on the Edinburg Postpartum Depression Scale (EDPS) used for this study may have evolved to full PPD, a fact that cannot be refuted owing to the one-staged sampling and short duration (February March) of the study. It is obvious also that it is not possible to assess period prevalence from this study. Comparing the two studies above exposes the reality of PPD and reveals a higher point prevalence of PPD among women in developing countries.

One clue to the public health implication of PPD, is its relationship with perinatal complications such as hospitalization during pregnancy and hospital admission of the baby. It is not clear if perinatal complications increase the risk of PPD 
or vice versa. In a Recent study [9], set out to examine whether specific pregnancy and delivery complications increased the risk for PPD. Using a prospective longitudinal study, researchers recruited a cohort of 4941 women and obtained data on perinatal complications from midwife/hospital registries and questionnaires and then assessed psychiatric symptoms 2 months after delivery using the standard EDPS scale. Logistic regression analysis was used to calculate risk. The study associated hospitalization during pregnancy, hospital admission of the baby, unplanned pregnancy, medical delivery and cesarean sections with increased risk of PPD. This study showed a great deal of statistical significance but it was not without flaws. One of its flaws, and a point of possible bias, was the heavy reliance on the diagnostic accuracy of midwife registries and on the ability of patients to recall symptoms. While the authors attempted to establish a temporal relationship by assessing delivery complications before assessing onset of PPD 2 months later, a point of concern that was omitted in the study was the baseline level of PPD at point of assessing for complications and whether perinatal complications always precede PPD since both can develop within the first weeks of delivery.

In another study [10], multivariate linear and logistic regression models were applied to a cohort of 1037 women to investigate the contribution of perinatal complications to PPD at 8 weeks postpartum. After adjusting for confounding factors, this study found that the contributions of factors such as hospitalization during pregnancy and unplanned pregnancy were explained by the socio-demographic characteristics of the mother. These studies appear to suggest a causal relationship between perinatal complications and PPD. This finding may not be generalizable to all perinatal complications because a recent study [11], found that the route of delivery (which reflects cesarean section and hospital admission rates) was non-predictive of PPD. The researchers in this study assessed the incidence and obstetric risk factors for PPD over 12 weeks after birth among Iranian women in primary health care centres (where the caesarean section rate is as high as $48 \%$ ). They were unable to conclusively associate route of delivery with PPD despite the use of a reliable diagnostic instrument. It is not clear if the exclusion of indigent and illiterate women or the general acceptance of operative delivery affected the outcomes of this study.

It is evident that there is not one study that provides a comprehensive evidence for the burden of postpartum depression. A more complete picture may be obtained by an in-depth meta-analysis of available evidence. So was the case of a study [12], looking into the epidemiology of PPD and its associated factors in Africa. It was a systematic review and meta-analysis of observational studies published in the 11 years preceding the study (2009 - 2020). The study pooled data of over 40,000 women and arrived at a pooled prevalence of $16.8 \%$ for PPD. It also identified low socioeconomic status and intimate partner violence as contributors to PPD. While the population and methodology of the study was well suited for its objectives, it was, like other previous studies, limited by publication bias because only articles published in English were included and only publications from 10 African countries were included. Its findings suggest a paucity of robust studies in Africa to produce generalizable evidence.

At least, from all the above, there is a general agreement that Postpartum depression is a global problem among mothers and the availability of standard tools for its diagnosis is well documented. Despite this, PPD remains under-detected and under-treated, posing serious implications for public health. To address the problems of PPD, first, there is a substantial need for additional research to identify the barriers to care and to women's preferences for care. This is especially necessary among low-income earners and minority populations who are at greater risk for PPD and yet are less likely to use treatment. There is a scarcity of generalizable evidence in the currently available data and experts must develop well-structured large scale, multi-centre studies to expose the full impact of PPD in the perinatal period. It is also necessary to define when to test for PPD, how often to test and in what setting to test in primary health care practice. Primary health care providers must establish a working relationship with their nursing staff, assistants and mental health providers that enables timely screening/diagnosis and effective referral of women with PPD for expert care.

Following expanded research, priority needs to be given to an effective mental health strategy within public health policy that provides an integrated care model for the prevention, detection and treatment of this condition in women [13]. Mandatory screening and reporting of PPD, increased awareness of the effects and risk factors for PPD, enabling access to rapid self-screening tools and support services for women may constitute a framework for an effective legislative approach to combating PPD [14]. This approach will depend on a system of continuity and sustainability in leadership at all levels and may suffer setbacks from variations in quality of healthcare leadership/delivery, geography and legislative styles with greater implications for low-income and indigent populations.

Considering the evidence for causality, and the multidirectional relationship between poverty, illiteracy, gender inequality, economic depravity and depressive disorders among women, a significant portion of the burden of mental disorders (to which PPD contributes significantly) among women, can be linked to persistent failures in attaining the Millennium Development Goals (). PPD is directly related to goals 4 (reduce child mortality) and 5 (to improve maternal health) of the since it imparts a woman's overall well-being, her ability to breastfeed and care for her baby and increases the rate of infanticide and suicide [15]. Efforts must then be made to remove the barriers to accelerated attainment of 
the. Some of the barriers, especially in under-developed countries, have been in the area of weak governance or health institutions, conflict/instability and environmental degradation. A robust approach to support developing countries must be explored to rapidly achieve these

\section{Conclusion}

Postpartum depression remains a serious burden among women of child-bearing age. Unfortunately, several decades since its first recognition, this burden has not been assessed with generalizable accuracy. In fact, researchers still struggle to recognize it as a distinct disease entity and no standard tool has been universally accepted for its diagnosis. The need for well structured, large scale multi-center studies and meta-analyses of current evidence is critical to obtain deeper insight into the magnitude of this burden and to develop strategies to tackling the problem of postpartum depression.

\section{Compliance with ethical standards}

\section{Disclosure of conflict of interest}

The Authors report no conflict of interest for the presented work.

\section{References}

[1] Shitu S, Geda B, Dheresa M. Postpartum depression and associated factors among mothers who gave birth in the last twelve months in Ankesha district, Awi zone, North West Ethiopia. BMC Preg Childbirth. 2019; $19: 485$.

[2] Bauman BL, Ko JY, Cox S. Vital Signs: Postpartum depressive symptoms and provider discussions about perinatal depression - United States. MMWR. 2018; 69: 575-581.

[3] Ghaedrahmati M, Kazemi A, Kheirabadi G, et al. Postpartum depression risk factors: A narrative review. J Educ Health Promt. 2017; 6: 60.

[4] Gebregziabher NK, Netsereab TB, Fessaha, YG. Prevalence and associated factors of postpartum depression among postpartum mothers in central region, Eritrea: a health facility based survey. BMC Pub Health. 2020; 20: 1614.

[5] Nonacs R, Cohen LS. Postpartum mood disorders: diagnosis and treatment guidelines. J Clin Psych. 1998; 59(2): 34-40.

[6] Cooper PJ, Tomlinson M, Swartz L, et al. Postpartum depression and the mother-infant relationship in a South African peri-urban settlement. British J Psych. 1999; 175: 554-8.

[7] Bobo WV, Yawn BP. Concise review for physicians and other clinicians: postpartum depression. Mayo Clin Proc. 2014; 89(6): 835-844.

[8] Almond P. Postnatal depression: a global public health perspective. Perspect Pub health. 2009; 129(5): $221-227$.

[9] Blom EA, Jansen PW, Verhulst FC, et al. Perinatal complications increase the risk of postpartum depression. The Generation R Study. BJOG. 2010; 117(11): 1390-1398.

[10] Koutra K, Vassilaki M, Georgiou V, et al. Pregnancy, perinatal and postpartum complications as determinants of postpartum depression: The Rhea mother-child cohort in Crete, Greece. J Epid Psych Sci. 2018; 27(3): $244-255$.

[11] Abdollahi F, Lye MS, Md Zain A, et al. Postnatal depression and its associated factors in women from different cultures. Iran J Psych Behav Sci. 2011; 5(2): 5-11.

[12] Dadi AF, Akalu TY, Baraki AG, et al. Epidemiology of postnatal depression and its associated factors in Africa: A systematic review and meta-analysis. PLoS ONE. 2020; 15(4): e0231940.

[13] Glavin K, Leahy-Warren P: Postnatal depression is a public health nursing issue: perspectives from norway and ireland. Nurs Research Prac. 2013; 8(1): 3409.

[14] Rhodes AM, Segre LS. Perinatal depression: a review of US legislation and law. Arch Womens Ment Health. 2013; 16(4): 259-270.

[15] Tsai AC, Tomlinson M. Mental health spillovers and the Millennium Development Goals: The case of perinatal depression in Khayelitsha, South Africa. J Glob Health. 2012; 2(1): 010302. 